\title{
A BOUNDARY VALUE PROBLEM OF ELASTOPLASTIC DEFORMATION PROCESS THEORY: EXISTENCE AND UNIQUENESS THEOREMS
}

\author{
DAO HUY BICH ${ }^{1}$
}

(Received 9 July 1991; revised 25 September 1992)

\begin{abstract}
This paper deals with the complete constitutive relations of elastoplastic deformation process theory, based on Ilyushin's postulate of isotropy and hypotheses of local determinancy and complanarity in plastic stage with complex loading. The formulation of the boundary value problem is given and existence and uniqueness theorems are considered.
\end{abstract}

\section{Introduction}

The analysis of stress-strain states or the stability of components or structures subjected to various complex loading beyond limits of elasticity requires a plasticity theory which can describe complex elastoplastic processes of deformation. The theory of elastoplastic deformation processes, based on Ilyushin's postulate of isotropy, satisfies this requirement. But up to now the stress-strain relationship has contained undetermined functionals. In recent years many papers have been published concerned with particular processes such as: simple loading process and process with small curvature; formulation and analysis of boundary value problems of these theories are studied. The general case is still open.

Assuming some approximation (hypothesis), we have constructed a complete stress-strain relationship of the local theory of elastoplastic deformation processes [4], based on Ilyushin's postulate of isotropy [5] and hypotheses of

\footnotetext{
${ }^{1}$ School of Mathematics and Statistics, Curtin University of Technology, Perth, Westem Australia. On leave from State University of Hanoi, Vietnam.

(C) Australian Mathematical Society, 1994, Serial-fee code 0334-2700/94
} 
local determinancy $[1,3,6,7,10]$ and complanarity [8]. These results can be applied to more general processes. The relationship for simple loading process, process with small and average curvature, unloading process and Prandtl - Reuss relations are considered as particular cases of this theory.

The formulation of the boundary value problem and analysis of the uniqueness theorem for plane deformation process are given in [3].

The aim of this paper is to analyse the stress-stain relationship and the solvability of the boundary value problem of local theory of elastoplastic deformation processes in general cases.

\section{Constitutive relations of local theory of elastoplastic deformation processes}

We shall employ the following notation:

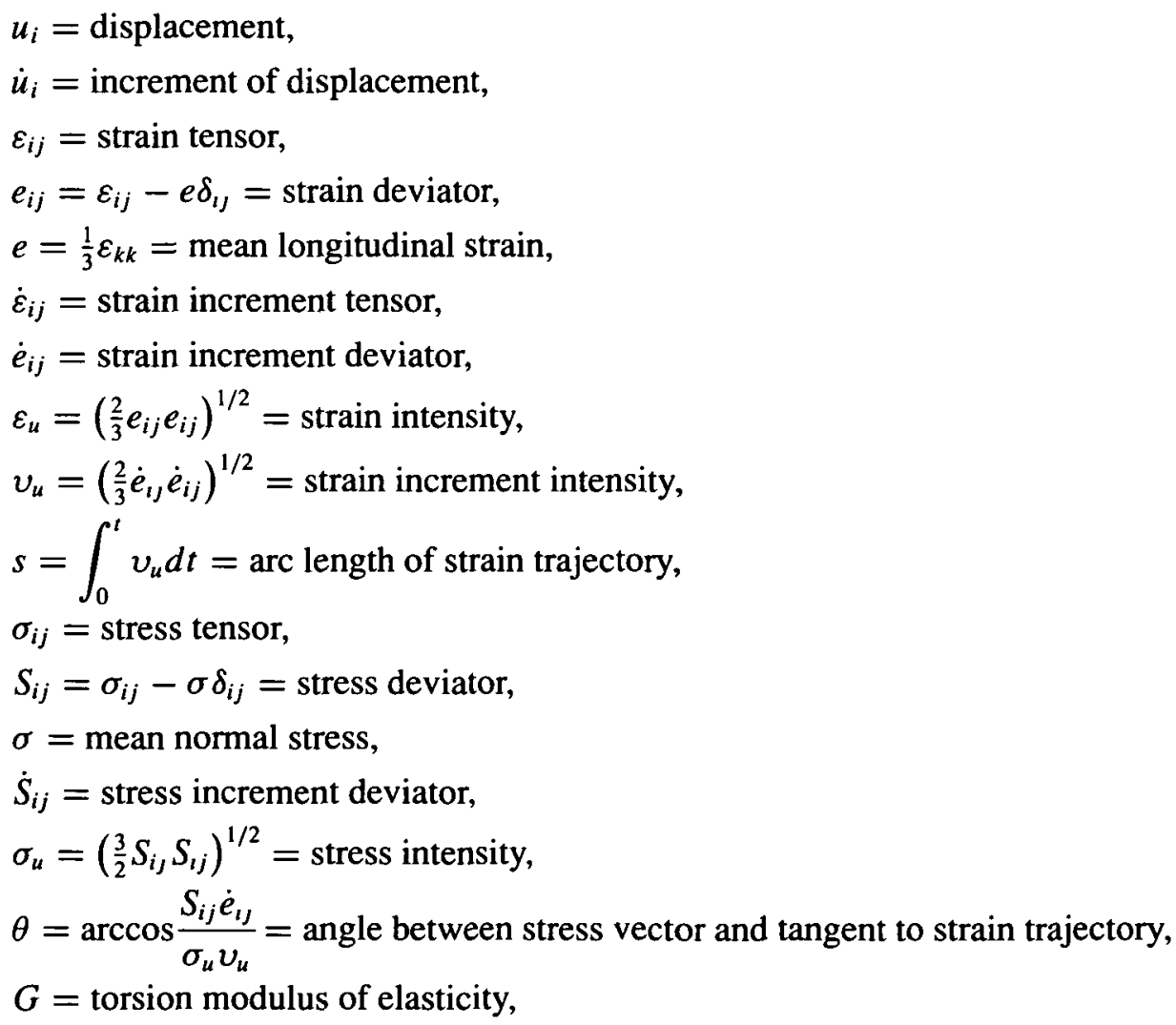


$K=$ compressive volume modulus of elasticity,

$f\left(\sigma_{u}, \theta, s\right), \psi(\theta, s)=$ material functions,

$\phi^{\prime}(s)=\frac{d \phi}{d s}=$ instantaneous slope of stress versus strain characteristic,

$\sigma_{s}=$ uniaxial yield stress,

$F_{i}=$ applied external surface force,

$\rho K_{i}=$ applied external volume force,

$\phi_{i}=$ given surface displacement,

$t=$ loading process parameter,

$x=$ radius vector of body's point,

$S=$ body surface,

$\Omega=$ body volume.

According to Ilyushin's postulate of isotropy [5] and hypotheses of local determinancy $[1,3,6,7,10]$ and complanarity of the stress vector, the stress increment vector and the strain increment vector [8], constitutive relations of the elastoplastic deformation process theory are of the form [4]:

$$
\begin{aligned}
\dot{S}_{i j} & =-\frac{2}{3} \frac{\sigma_{u} f}{\sin \theta} \dot{e}_{i j}+\left(\frac{\psi}{\cos \theta}+\frac{\sigma_{u} f}{\sin \theta}\right) \frac{S_{m n} \dot{e}_{m n}}{\sigma_{u}^{2}} S_{i j}, \\
\dot{\sigma} & =3 K \dot{e},
\end{aligned}
$$

where

$$
\begin{gathered}
f \equiv f\left(\sigma_{u}, \theta, s\right)=-\frac{1}{s} \sin \theta\left[1+\left(\frac{3 G_{s}}{\sigma_{u}}-1\right)\left(\frac{1-\cos \theta}{2}\right)^{\alpha}\right], \\
\psi \equiv \psi(\theta, s)=\phi^{\prime}(s) \cos \theta-\left(3 G-\phi^{\prime}\right)\left(\frac{1-\cos \theta}{2}\right)^{\beta}, \\
\alpha \geq 1, \quad \beta>1, \quad 0 \leq \theta \leq \pi .
\end{gathered}
$$

$\alpha$ and $\beta$ are constants, depending upon the material being used.

It is significant that $f$ and $\psi$ can be applied for all active and passive deformation processes, i.e. the stress-strain relationship (1.1) - (1.2) can describe all deformation processes with complex loading (not only loading, but unloading as well). Material functions $f$ and $\psi$, depending upon the materials used, are approximated analytically in the form (1.2) based on experimental data [4, 2]. The stress-strain relations for plane deformation process have been considered in $[1,3,11]$. 


\section{Particular cases:}

\section{A. SIMPLE LOADING PROCESS}

For this process $\theta=0$,

$$
\lim _{\theta \rightarrow 0} \frac{f}{\sin \theta}=-\frac{1}{s}, \quad \lim _{\theta \rightarrow 0} \psi=\phi^{\prime}(s) ;
$$

the relations (1.1) - (1.2) become

$$
\dot{S}_{i j}=\frac{2}{3} \frac{\sigma_{u}}{s} \dot{e}_{i j}+\left(\phi^{\prime}-\frac{\sigma_{u}}{s}\right) \frac{S_{i j}}{\sigma_{u}} v_{u} .
$$

Otherwise, according to the small elastoplastic deformation theory for simple loading

$$
S_{i j}=\frac{2}{3} \frac{\sigma_{u}}{\varepsilon_{u}} e_{i j}
$$

we obtain

$$
\dot{S}_{i j}=\frac{2}{3} \frac{\sigma_{u}}{\varepsilon_{u}} \dot{e}_{i j}+\frac{2}{3}\left(\frac{1}{\varepsilon_{u}} \frac{d \sigma_{u}}{d \varepsilon_{u}}-\frac{\sigma_{u}}{\varepsilon_{u}^{2}}\right) \dot{\varepsilon}_{u} e_{i j} .
$$

Substituting $e_{i j}$ from (1.4) gives

$$
\dot{S}_{i j}=\frac{2}{3} \frac{\sigma_{u}}{\varepsilon_{u}} \dot{e}_{i j}+\left(\frac{d \sigma_{u}}{d \varepsilon_{u}}-\frac{\sigma_{u}}{\varepsilon_{u}}\right) \frac{S_{i j}}{\sigma_{u}} \dot{\varepsilon}_{u} .
$$

Since a simple loading process always gives

$$
s=\varepsilon_{u}, \quad \dot{s}=\dot{\varepsilon}_{u}=v_{u}, \quad \frac{d \sigma_{u}}{d \varepsilon_{u}}=\frac{d \sigma_{u}}{d s}=\phi^{\prime}(s) ;
$$

relation (1.5) reduces to (1.3).

In the elastic stage, the material displays elastic behaviour until yield occurs $\sigma_{u} \leq \sigma_{s}$. The stress versus strain characteristic is a straight line with slope $3 G$, so that $\sigma_{u} / s=3 G, \phi^{\prime}=3 G$. Hence the relations (1.3) or (1.5) reduces to a simple Hookean relationship of the form

$$
\dot{S}_{i j}=2 G \dot{e}_{i j} \text {. }
$$

\section{B. UNLOADING PROCESS}

The unloading process occurs when $\theta=\pi$, i.e. the direction of the tangent to the continuing deformation trajectory is opposite to the stress vector at considered point. Since

$$
\lim _{\theta \rightarrow \pi} \frac{f}{\sin \theta}=-\frac{3 G}{\sigma_{u}}, \quad \lim _{\theta \rightarrow \pi} \psi=-3 G
$$


(1.1) - (1.2) become

$$
\dot{S}_{i j}=2 G \dot{e}_{i j}
$$

C. DEFORMATION PROCESS WITH AVERAGE CURVATURE

For this process the value of angle $\theta$ is small. Restricting to the second-order small values we obtain, from (1.2),

$$
\begin{aligned}
& f=-\frac{1}{s} \sin \theta \approx-\frac{\theta}{s}, \\
& \psi=\phi^{\prime} \cos \theta \approx \phi^{\prime}\left(1-\theta^{2} / 2\right) .
\end{aligned}
$$

Substituting into (1.1) - (1.2) gives

$$
\dot{S}_{i j}=\frac{2}{3} \frac{\sigma_{u}}{s} \dot{e}_{i j}+\left(\phi^{\prime}-\frac{\sigma_{u}}{s}\right) \frac{S_{m n} \dot{e}_{m n}}{\sigma_{u}^{2}} S_{i j} .
$$

The relation (1.8) is a generalization of Prandtl-Reuss relation for perfectly plastic material and Prager relations for plastic strain-hardening material.

\section{Boundary value problem of local theory of elastoplastic deformation processes}

Let $K_{l}(x, t)$ and $F_{i}(x, t)$ be external volume and surface forces that act on the body and let $\varphi_{i}(x, t)$ be displacement on the body's surface. It is necessary to find displacements $u_{i}(x, t) \in C^{2}(\Omega) \cap C^{1}(\bar{\Omega})$, strain tensor $\varepsilon_{i j}(x, t)$ and stress tensor $\sigma_{i j}(x, t) \in C^{1}(\Omega) \cap C^{0}(\bar{\Omega})$, where $\bar{\Omega}=\Omega \cup S, t \in[0, T]$, that satisfy the following equations:

$$
\begin{aligned}
& \frac{\partial \sigma_{i j}}{\partial x_{j}}+\rho K_{i}=0 \quad x \in \Omega, \\
& \varepsilon_{i j}=\frac{1}{2}\left(\frac{\partial u_{i}}{\partial x_{j}}+\frac{\partial u_{j}}{\partial x_{i}}\right) \quad x \in \bar{\Omega}, \\
& \dot{S}_{i j}=-\frac{2}{3} \frac{\sigma_{u} f}{\sin \theta} \dot{e}_{i j}+\left(\frac{\psi}{\cos \theta}+\frac{\sigma_{u} f}{\sin \theta}\right) \frac{S_{m n} \dot{e}_{m n}}{\sigma_{u}^{2}} S_{i j} \quad x \in \bar{\Omega}, \\
& \dot{\sigma}=3 K \dot{e},
\end{aligned}
$$

and the boundary conditions

$$
\begin{aligned}
\sigma_{i j} n_{J} & =F_{i} \quad x \in S_{\sigma}, \\
u_{i} & =\varphi_{i} \quad x \in S_{u}, \\
S_{\sigma} \cup S_{u} & =S, \quad S_{\sigma} \cap S_{u}=\phi \quad t \in[0, T] .
\end{aligned}
$$


A boundary problem formulated in this way is called a 'global' problem. The problem now can be formulated also 'in small', i.e. with displacement, strain and stress increments at any fixed $t=t_{o}$.

Let $\dot{K}_{i}\left(x, t_{o}\right), \dot{F}_{i}\left(x, t_{o}\right)$ be given increments of external forces and let $\dot{\varphi}_{i}\left(x, t_{o}\right)$ denote the increment of displacement on the body's surface. It is necessary to find increments

$$
\dot{u}_{i}\left(x, t_{o}\right), \quad \dot{\varepsilon}_{i j}\left(x, t_{o}\right), \quad \dot{\sigma}_{i j}\left(x, t_{o}\right)
$$

that satisfy the following equations:

$$
\begin{aligned}
\frac{\partial \dot{\sigma}_{i j}\left(x, t_{o}\right)}{\partial x}+ & \rho \dot{K}_{i}\left(x, t_{o}\right)=0 \quad x \in \Omega, \\
\dot{\varepsilon}_{i j}\left(x, t_{o}\right)= & \frac{1}{2}\left(\frac{\partial \dot{u}_{i}\left(x, t_{o}\right)}{\partial x_{j}}+\frac{\partial \dot{u}_{j}\left(x, t_{o}\right)}{\partial x_{i}}\right) \quad x \in \bar{\Omega}, \\
\dot{S}_{i j}\left(x, t_{o}\right)= & -\frac{2}{3} \frac{\sigma_{u o} f_{o}\left(\sigma_{u o}, \theta_{o}, s_{o}\right)}{\sin \theta_{o}} \dot{e}_{i j}\left(x, t_{o}\right) \\
& +\left(\frac{\psi_{o}\left(\theta_{o}, s_{o}\right)}{\cos \theta_{o}}+\frac{\sigma_{u o} f_{o}}{\sin \theta_{o}}\right) \frac{S_{m n}\left(x, t_{o}\right) \dot{e}_{m n}\left(x, t_{o}\right)}{\sigma_{u o}^{2}} S_{i j}\left(x, t_{o}\right), \\
\dot{\sigma}\left(x, t_{o}\right)= & 3 K \dot{e}\left(x, t_{o}\right) \quad x \in \bar{\Omega},
\end{aligned}
$$

and the boundary conditions

$$
\begin{aligned}
\dot{\sigma}_{i j}\left(x, t_{o}\right) n_{j} & =\dot{F}_{i}\left(x, t_{o}\right) \quad x \in S_{\sigma}, \\
\dot{u}_{i}\left(x, t_{o}\right) & =\phi_{l}\left(x, t_{o}\right) \quad x \in S_{u},
\end{aligned}
$$

where

$$
\begin{aligned}
\theta_{o} & =\arccos \frac{S_{i j}\left(x, t_{o}\right) \dot{e}_{i j}\left(x, t_{o}\right)}{\sigma_{u o} v_{u o}} \\
\sigma_{u o} & =\sigma_{u}\left(x, t_{o}\right)=\left[\frac{3}{2} S_{i j}\left(x, t_{o}\right) S_{i j}\left(x, t_{o}\right)\right]^{1 / 2}, \\
v_{u o} & =v_{u}\left(x, t_{o}\right)=\left[\frac{2}{3} \dot{e}_{i j}\left(x, t_{o}\right) \dot{e}_{i j}\left(x, t_{o}\right)\right]^{1 / 2}
\end{aligned}
$$

Combining (2.8) - (2.10) we can express $\dot{\sigma}_{i j}$ as functions of $\dot{u}_{t}$, or shortly $\dot{\sigma}_{i j}(u)$. Substituting into (2.7) gives

$$
\frac{\partial \dot{\sigma}_{i j}(u)}{\partial x_{j}}+\rho \dot{K}_{t}=0 .
$$


Let $v$ be any smooth vector function such that

$$
v \equiv\left\{v_{i}\left(x, t_{o}\right)\right\} \quad \text { and } \quad v_{i}\left(x, t_{o}\right)=0 \quad \text { on } S_{u} .
$$

Multiplying (2.13) by $v_{i}$ and integrating over the entire volume $\Omega$ of the body, we obtain

$$
\begin{aligned}
\int_{\Omega}\left(\frac{\partial \dot{\sigma}_{t}(u)}{\partial x_{j}}+\rho \dot{K}_{i}\right) v_{i} d \Omega & =\int_{\Omega}\left[\frac{\partial\left(\dot{\sigma}_{i j}(u) v_{\imath}\right)}{\partial x_{j}}-\dot{\sigma}_{i j}(u) \frac{\partial v_{i}}{\partial x_{j}}\right] d \Omega+\int_{\Omega} \rho \dot{K}_{t} v_{\imath} d \Omega \\
& =0 .
\end{aligned}
$$

Using the divergence theorem and the boundary conditions $(2.11),(2.12)$ we have:

$$
\begin{aligned}
& \int_{\Omega} \dot{\sigma}_{i j}(u) \dot{\varepsilon}_{i j}(v) d \Omega=\int_{\Omega} \rho \dot{K}_{i} v_{l} d \Omega+\int_{S_{s}} \dot{F}_{i} v_{i} d S \\
& \forall v: v \equiv\left\{v_{i}\right\},\left.\quad v\right|_{s_{u}}=0,
\end{aligned}
$$

where

$$
\dot{\varepsilon}_{i j}(v)=\frac{1}{2}\left(\frac{\partial v_{i}}{\partial x_{j}}+\frac{\partial v_{j}}{\partial x_{i}}\right)
$$

The equation (2.14) can be written in the form

$$
\int_{\Omega} \dot{S}_{l j}(u) \dot{e}_{i j}(v) d \Omega+3 \int_{\Omega} \dot{\sigma}(u) \dot{e}(v) d \Omega=\int_{\Omega} \rho \dot{K}_{i} v_{i} d \Omega+\int_{\substack{S_{\sigma} \\ \forall v,}} \dot{F}_{i} v_{i} d S
$$

The boundary value problem can be formulated either in the form (2.7) (2.13), or in the form (2.15).

\section{Existence and uniqueness theorems for the boundary value problem}

The vector function $u$ is to be called a generalized solution of the boundary value problem (2.7) - (2.12), if $u$ satisfies (2.8), constitutive relations (2.9), (2.10) and identity (2.15) for all $v$ such that $\left.v\right|_{S_{u}}=0$. The function $v$ is not required to be smooth. If the generalized solution is smooth enough, then it is a classical solution of the problem (2.7) - (2.12).

We shall consider the function $u$ as an element of the functional space $H(\Omega)$ with the norm

$$
\|u\|_{H}^{2}=\int_{\Omega}|u|^{2} d \Omega+\int_{\Omega} \frac{\partial u}{\partial x_{i}} \cdot \frac{\partial u}{\partial x_{i}} d \Omega .
$$


Since

$$
\int_{\Omega}|u|^{2} d \Omega \leq C_{1} \int_{\Omega} \frac{\partial u}{\partial x_{i}} \cdot \frac{\partial u}{\partial x_{i}} d \Omega, \quad C_{1}=\text { const }
$$

and

$$
\int_{\Omega} \frac{\partial u}{\partial x_{i}} \cdot \frac{\partial u}{\partial x_{i}} d \Omega \leq C_{2} \int_{\Omega}\left(\frac{\partial \dot{u}_{i}}{\partial x_{j}}+\frac{\partial \dot{u}_{j}}{\partial x_{i}}\right)\left(\frac{\partial \dot{u}_{i}}{\partial x_{j}}+\frac{\partial \dot{u}_{j}}{\partial x_{i}}\right) d \Omega, \quad C_{2}=\text { const }
$$

the norm becomes:

$$
\|u\|_{H}^{2}=\int_{\Omega} \dot{\varepsilon}_{i j}(u) \dot{\varepsilon}_{i j}(u) d \Omega=\int_{\Omega} \dot{e}_{i j}(u) \dot{e}_{i j}(u) d \Omega+3 \int_{\Omega} \dot{e}^{2}(u) d \Omega .
$$

Now consider the functional

$$
I(u, v)=\int_{\Omega} \dot{\sigma}_{i j}(u) \dot{\varepsilon}_{i j}(v) d \Omega .
$$

The functional $I$ is linear because of the linearity of $\dot{\varepsilon}_{i j}(v)$ with respect to $v$. In order to prove the continuity of $I$, it is necessary to show that

$$
\int_{\Omega} \dot{\sigma}_{i j}(u) \dot{\varepsilon}_{i j}(v) d \Omega \leq M\|u\|_{H}\|v\|_{H}, \quad M<+\infty .
$$

In fact we have

$$
\begin{aligned}
\int_{\Omega} \dot{\sigma}_{i j}(u) \dot{\varepsilon}_{i j}(v) d \Omega & =\int_{\Omega} \dot{S}_{i j}(u) \dot{e}_{i j}(v) d \Omega+3 \int_{\Omega} \dot{\sigma}(u) \dot{e}(v) d \Omega \\
\leq & \left(\int_{\Omega} \dot{S}_{i j}(u) \dot{S}_{l j}(u) d \Omega\right)^{1 / 2}\left(\int_{\Omega} \dot{e}_{i j}(v) \dot{e}_{i j}(v) d \Omega\right)^{1 / 2} \\
& +9 K\left(\int_{\Omega} \dot{e}^{2}(u) d \Omega\right)^{1 / 2}\left(\int_{\Omega} \dot{e}^{2}(v) d \Omega\right)^{1 / 2}
\end{aligned}
$$

According to (2.9), we have

$$
\begin{aligned}
& \dot{S}_{i j}(u) \dot{S}_{i j}(u)= {\left[-\frac{2}{3} \frac{\sigma_{u o} f_{o}}{\sin \theta_{o}} \dot{e}_{i j}(u)+\left(\frac{\psi_{o}}{\cos \theta_{o}}+\frac{\sigma_{u o} f_{o}}{\sin \theta_{o}}\right) \frac{S_{m n} \dot{e}_{m n}}{\sigma_{u o}^{2}} S_{i j}\right] } \\
& \times\left[-\frac{2}{3} \frac{\sigma_{u o} f_{o}}{\sin \theta_{o}} \dot{e}_{i j}(u)+\left(\frac{\psi_{o}}{\cos \theta_{o}}+\frac{\sigma_{u o} f_{o}}{\sin \theta_{o}}\right) \frac{S_{m n} \dot{e}_{m n}}{\sigma_{u o}^{2}} S_{i j}\right] \\
&=\frac{2}{3} v_{u o}^{2}\left(\sigma_{u o}^{2} f_{o}^{2}+\psi_{o}^{2}\right) \\
&=\frac{4}{9} \dot{e}_{i j}(u) \dot{e}_{i j}(u)\left(\sigma_{u o}^{2} f_{o}^{2}+\psi_{o}^{2}\right) .
\end{aligned}
$$


Hence

$$
\begin{gathered}
\int_{\Omega} \dot{\sigma}_{i j}(u) \dot{\varepsilon}_{i j}(v) d \Omega \leq\left(\int_{\Omega} \frac{4}{9}\left(\sigma_{u o}^{2} f_{o}^{2}+\psi_{o}^{2}\right) \dot{e}_{i j}(u) \dot{e}_{i j}(u) d \Omega\right)^{1 / 2}\left(\int_{\Omega} \dot{e}_{i j}(v) \dot{e}_{i j}(v) d \Omega\right)^{1 / 2} \\
+3 K\left(\int_{\Omega} 3 \dot{e}^{2}(u) d \Omega\right)^{1 / 2}\left(\int_{\Omega} 3 \dot{e}^{2}(v) d \Omega\right)^{1 / 2} \\
\leq M\left[\left(\int_{\Omega} \dot{e}_{i j}(u) \dot{e}_{i j}(u) d \Omega\right)^{1 / 2}\left(\int_{\Omega} \dot{e}_{i j}(v) \dot{e}_{i j}(v) d \Omega\right)^{1 / 2}\right. \\
\left.+\left(\int_{\Omega} 3 \dot{e}^{2}(u) d \Omega\right)^{1 / 2}\left(\int_{\Omega} 3 \dot{e}^{2}(v) d \Omega\right)^{1 / 2}\right]
\end{gathered}
$$

where

$$
M=\max \left\{\frac{2}{3}\left(\sigma_{u o}^{2} f_{o}^{2}+\psi_{o}^{2}\right)^{1 / 2} ; 3 K\right\}<+\infty .
$$

Applying the Cauchy-Schwarz inequality, we can rewrite the above as follows:

$$
\begin{aligned}
\int_{\Omega} \dot{\sigma}_{i j}(u) \dot{\varepsilon}_{i j}(v) d \Omega \leq & M\left(\int_{\Omega} \dot{e}_{i j}(u) \dot{e}_{i j}(u) d \Omega+\int_{\Omega} 3 \dot{e}^{2}(u) d \Omega\right)^{1 / 2} \\
& \left(\int_{\Omega} \dot{e}_{i j}(v) \dot{e}_{i j}(v) d \Omega+\int_{\Omega} 3 \dot{e}^{2}(v) d \Omega\right)^{1 / 2} \\
& =M\|u\|_{H}\|v\|_{H} .
\end{aligned}
$$

Therefore $I$ is linear and continuous on $H(\Omega)$. It follows from Riesz's theorem that there exists an operator $A: H(\Omega) \rightarrow H^{*}(\Omega)$, where $H^{*}(\Omega)$ is the dual functional space of $H(\Omega)$, such that:

$$
(A u, v)_{H}=\int_{\Omega} \dot{\sigma}_{i j}(u) \dot{\varepsilon}_{i j}(v) d \Omega .
$$

The operator $A$ is called a fundamental operator of the boundary value problem. If

$$
\rho \dot{K}_{i} \in L_{p}, \quad p \geq 6 / 5, \quad \dot{F}_{1} \in L_{q}, \quad q>4 / 3,
$$

then the expression at the right hand side of (2.15) is also a linear continuous functional on $H(\Omega)$, and there exists an operator $L: H \rightarrow H^{*}$ such that:

$$
\int_{\Omega} \rho \dot{K}_{i} v_{i} d \Omega+\int_{S_{\sigma}} \dot{F}_{i} v_{i} d S=(L, v)_{H}
$$


The equation (2.15) reduces to an equivalent operator equation

$$
A u=L \quad u \in H(\Omega)
$$

A generalized solution of the boundary value problem (2.7) - (2.12) is also a solution of the operator equation (3.6) and conversely.

In order to prove existence and uniqueness of the solution of the boundary value problem we shall consider properties of the operator $A$.

a. OPERATOR $A$ IS COERCIVE

$$
(A u, u)_{H} \geq \gamma\|u\|_{H}^{2} ; \quad \gamma>0 ; \quad \forall u \in H(\Omega) .
$$

PROOF.

$$
\begin{aligned}
(A u, u)_{H}= & \int_{\Omega}\left[-\frac{2}{3} \frac{\sigma_{u o} f_{o}}{\sin \theta_{o}} \dot{e}_{i j}(u)+\left(\frac{\psi_{o}}{\sin \theta_{o}}+\frac{\sigma_{u o} f_{o}}{\sin \theta_{o}}\right) \frac{S_{m n} \dot{e}_{m n}(u)}{\sigma_{u o}^{2}} S_{i j}\right] \dot{e}_{i j}(u) d \Omega \\
& +9 K \int_{\Omega} \dot{e}^{2}(u) d \Omega \\
\geq & \gamma\left[\int_{\Omega} \dot{e}_{i j}(u) \dot{e}_{i j}(u) d \Omega+3 \int_{\Omega} \dot{e}^{2}(u) d \Omega\right] \\
= & \gamma\|u\|_{H}^{2},
\end{aligned}
$$

where

$$
\gamma=\min \left\{3 K ; \frac{2}{3}\left(\psi_{o} \cos \theta_{o}-\sigma_{u o} f_{o} \sin \theta_{o}\right)\right\}
$$

or

$$
\frac{2}{3}\left(\psi_{o} \cos \theta_{o}-\sigma_{u o} f_{o} \sin \theta_{o}\right) \geq \gamma>0, \quad 0 \leq \theta_{o} \leq \pi .
$$

Hence, if condition (3.7) is satisfied, then the operator $A$ is coercive. Later condition (3.7) can be verified with $f_{0}, \psi_{0}$ indicated in (1.2).

b. OPERATOR A IS STRICTLY MONOTONE

$$
\left(A u_{1}-A u_{2}, u_{1}-u_{2}\right)_{H} \geq 0, \quad \forall u_{1}, u_{2} \in H(\Omega) .
$$

Introducing the five-dimensional strain vector $\ni$ and stress vector $\sigma$, with components defined in the form 


$$
\begin{aligned}
\ni: \quad \vartheta_{1} & =e_{11}, \quad \partial_{2}=\frac{1}{\sqrt{3}}\left(e_{11}+2 e_{22}\right), \quad \ni_{3}=\frac{2}{\sqrt{3}} e_{12}, \\
\vartheta_{4} & =\frac{2}{\sqrt{3}} e_{23}, \quad \ni_{5}=\frac{2}{\sqrt{3}} e_{31} ; \\
|\ni|^{2} & =\ni_{i} \ni_{i}=\varepsilon_{u}^{2}, \\
\sigma: \quad \sigma_{1} & =\frac{3}{2} S_{11}, \quad \sigma_{2}=\frac{\sqrt{3}}{2}\left(S_{11}+2 S_{22}\right), \quad \sigma_{3}=\sqrt{3} S_{12}, \\
\sigma_{4} & =\sqrt{3} S_{23}, \quad \sigma_{5}=\sqrt{3} S_{31} ; \\
|\sigma|^{2} & =\sigma_{i} \sigma_{i}=\sigma_{u}^{2},
\end{aligned}
$$

we rewrite the expression at the left hand side in terms of stress and strain vectors as

$$
\left(A u_{1}-A u_{2}, u_{1}-u_{2}\right)_{H}=\int_{\Omega}\left(\dot{\sigma}_{1}-\dot{\sigma}_{2}\right)\left(\dot{\Xi}_{1}-\dot{\Xi}_{2}\right) d \Omega+9 K \int_{\Omega}\left(\dot{e}_{1}-\dot{e}_{2}\right)^{2} d \Omega .
$$

The stress-strain relation (2.9) now can be written in the form

$$
\dot{\boldsymbol{\sigma}}=-\frac{\sigma_{u o} f_{o}}{\sin \theta_{o}} \dot{\boldsymbol{g}}+\left(\frac{\psi_{o}}{\cos \theta_{o}}+\frac{\sigma_{u o} f_{o}}{\sin \theta_{o}}\right) \frac{\boldsymbol{\sigma} \cdot \dot{\boldsymbol{\partial}}}{\sigma_{u o}^{2}} \boldsymbol{\sigma} .
$$

Introducing the unit vector $n$, perpendicular to $\sigma / \sigma_{u o}$ and situated on the plane containing vectors $\sigma / \sigma_{u}$ and $\dot{\boldsymbol{\xi}}$, we can express the vector $\dot{\boldsymbol{\partial}}$ as

$$
\dot{\partial}=v_{u o} \cos \theta_{o} \frac{\boldsymbol{\sigma}}{\sigma_{u o}} \pm v_{u o} \sin \theta_{o} \boldsymbol{n}
$$

i.e. $\boldsymbol{n}$ is apparently chosen to ensure a decomposition of the strain-rate vector.

The stress-strain relation (2.9) becomes

$$
\dot{\boldsymbol{\sigma}}=v_{u o} \psi_{o} \frac{\boldsymbol{\sigma}}{\sigma_{u o}} \mp \sigma_{u o} v_{u o} f_{o} n \text {. }
$$

Define $k=\sigma / \sigma_{u o}$ (with $t=t_{o}, k$ is fixed),

$$
\begin{aligned}
\xi & =v_{u o} \cos \theta_{o}, \quad \eta=v_{u o} \sin \theta_{o} \\
P & =v_{u o} \psi_{o}\left(\theta_{o}, s_{o}\right) \equiv P\left(\xi, \eta, s_{o}\right) \\
Q & =-\sigma_{u o} v_{u o} f_{o}\left(\theta_{o}, \sigma_{u o}, s_{o}\right) \equiv Q\left(\xi, \eta, \sigma_{u o}, s_{o}\right)
\end{aligned}
$$

and rewrite (3.9), (3.10) in the form:

$$
\dot{\boldsymbol{\Xi}}_{1}=\xi_{1} k \pm \eta_{1} n_{1} \quad \dot{\sigma}_{1}=P\left(\xi_{1}, \eta_{1}, s_{o}\right) k \pm Q\left(\xi_{1}, \eta_{1}, \sigma_{u o}, s_{o}\right) n_{1}
$$


and

$$
\dot{\ni}_{2}=\xi_{2} k \pm \eta_{2} n_{2} \quad \dot{\sigma}_{2}=P\left(\xi_{2}, \eta_{2}, s_{o}\right) k \pm Q\left(\xi_{2}, \eta_{2}, \sigma_{u o}, s_{o}\right) n_{2}
$$

Vector $\boldsymbol{k}$ is fixed, but vector $\boldsymbol{n}$ is changed, depending on $\dot{\boldsymbol{\partial}}_{1}, \dot{\boldsymbol{\partial}}_{2}$. In general $\dot{\boldsymbol{\partial}}_{1}, \dot{\boldsymbol{\partial}}_{2}$ do not lie on the same plane, containing $\boldsymbol{k}$ and $\boldsymbol{n}$, so that two planes $\left(\boldsymbol{k}, \boldsymbol{n}_{1}\right)$ and $\left(\boldsymbol{k}, \boldsymbol{n}_{2}\right)$ do not coincide.

Substituting into (3.8) gives

$$
\begin{aligned}
\left(A u_{1}-A u_{2}, u_{1}-u_{2}\right)_{H}=\int_{\Omega}\{ & \left.P\left(\xi_{1}, \eta_{1}, s_{o}\right)-P\left(\xi_{2}, \eta_{2}, s_{o}\right)\right]\left(\xi_{1}-\xi_{2}\right) \\
& +Q\left(\xi_{1}, \eta_{1}, \sigma_{u o}, s_{o}\right) \eta_{1}+Q\left(\xi_{2}, \eta_{2}, \sigma_{u o}, s_{o}\right) \eta_{2} \\
& \left.-n_{1} \cdot n_{2}\left[Q\left(\xi_{1}, \eta_{1}, \ldots\right) \eta_{2}+Q\left(\xi_{2}, \eta_{2}, \ldots\right) \eta_{1}\right]\right\} d \Omega \\
+ & 9 K \int_{\Omega}\left(\dot{e}_{1}-\dot{e}_{2}\right)^{2} d \Omega .
\end{aligned}
$$

Since $\eta=v_{u o}, \sin \theta_{o} \geq 0$ and

$$
Q=-\sigma_{u o} v_{u o} f_{o}=\frac{1}{s_{o}} \sigma_{u o} v_{u o}\left[1+\left(\frac{3 G s_{o}}{\sigma_{u o}}-1\right)\left(\frac{1-\cos \theta_{o}}{2}\right)^{\alpha}\right] \sin \theta_{o} \geq 0
$$

for all $\theta_{o}$, where $0 \leq \theta_{o} \leq \pi ;\left|\boldsymbol{n}_{1} \cdot \boldsymbol{n}_{2}\right| \leq 1$, so that

$$
\begin{aligned}
& {\left[P\left(\xi_{1}, \eta_{1}, \ldots\right)-P\left(\xi_{2}, \eta_{2}, \ldots\right)\right]\left(\xi_{1}-\xi_{2}\right)} \\
& +Q\left(\xi_{1}, \eta_{1}, \ldots\right) \eta_{1}+Q\left(\xi_{2}, \eta_{2}, \ldots\right) \eta_{2} \\
& -n_{1} \cdot n_{2}\left[Q\left(\xi_{1}, \eta_{1}, \ldots\right) \eta_{2}+Q\left(\xi_{2}, \eta_{2}, \ldots\right) \eta_{1}\right] \\
& \quad \geq\left[P\left(\xi_{1}, \eta_{1}, \ldots\right)-P\left(\xi_{2}, \eta_{2}, \ldots\right)\right]\left(\xi_{1}-\xi_{2}\right) \\
& \quad+\left[Q\left(\xi_{1}, \eta_{1}, \ldots\right)-Q\left(\xi_{2}, \eta_{2}, \ldots\right)\right]\left(\eta_{1}-\eta_{2}\right) .
\end{aligned}
$$

Using the mean value theorem we obtain

$$
\begin{aligned}
& {\left[P\left(\xi_{1}, \eta_{1}, \ldots\right)-P\left(\xi_{2}, \eta_{2}, \ldots\right)\right]\left(\xi_{1}-\xi_{2}\right)} \\
& +\left[Q\left(\xi_{1}, \eta_{1}, \ldots\right)-Q\left(\xi_{2}, \eta_{2}, \ldots\right)\right]\left(\eta_{1}-\eta_{2}\right) \\
& =\left.\frac{\partial P}{\partial \xi}\right|_{\xi^{*}, \eta^{*}}\left(\xi_{1}-\xi_{2}\right)^{2} \\
& +\left[\left.\frac{\partial P}{\partial \eta}\right|_{\xi^{*}, \eta^{*}}+\left.\frac{\partial Q}{\partial \xi}\right|_{\xi^{* *,}, \eta^{* *}}\right]\left(\xi_{1}-\xi_{2}\right)\left(\eta_{1}-\eta_{2}\right) \\
& +\left.\frac{\partial Q}{\partial \eta}\right|_{\xi^{* *}, \eta^{* *}}\left(\eta_{1}-\eta_{2}\right)^{2}
\end{aligned}
$$


where $\xi^{*}, \xi^{* *} \in\left[\xi_{1}, \xi_{2}\right], \eta^{*}, \eta^{* *} \in\left[\eta_{1}, \eta_{2}\right]$.

This expression must be positive, i.e. the quadratic form must be positive definite, so that

$$
\begin{gathered}
\frac{\partial P\left(\xi^{*}, \eta^{*}, s_{o}\right)}{\partial \xi}>0, \\
4 \frac{\partial P\left(\xi^{*}, \eta^{*}, s_{o}\right)}{\partial \xi} \frac{\partial Q\left(\xi^{* *}, \eta^{* *}, \sigma_{u o}, s_{o}\right)}{\partial \eta} \\
-\left[\frac{\partial P\left(\xi^{*}, \eta^{*}, s_{o}\right)}{\partial \eta}+\frac{\partial Q\left(\xi^{* *}, \eta^{* *}, \sigma_{u o}, s_{o}\right)}{\partial \xi}\right]^{2}>0 .
\end{gathered}
$$

With the quantities $P$ and $Q$ as shown above, these conditions become

$$
\begin{gathered}
\psi_{o}\left(\theta_{o}^{*}, s_{o}\right) \cos \theta_{o}^{*}-\frac{\partial \psi_{o}\left(\theta_{o}^{*}, s_{o}\right)}{\partial \theta_{o}} \sin \theta_{o}^{*}>0 \\
4 \sigma_{u o}\left[\frac{\partial \psi_{o}\left(\theta_{o}^{*}, s_{o}\right)}{\partial \theta_{o}} \sin \theta_{o}^{*}-\psi_{o}\left(\theta_{o}^{*}, s_{o}\right) \cos \theta_{o}^{*}\right] \\
\times\left[\frac{\partial f_{o}\left(\theta_{o}^{* *}, \sigma_{u o}, s_{o}\right)}{\partial \theta_{o}} \cos \theta_{o}^{* *}+f_{o}\left(\theta_{o}^{* *}, \sigma_{u o}, s_{o}\right) \sin \theta_{o}^{* *}\right] \\
-\left[\left(\frac{\partial \psi_{o}\left(\theta_{o}^{*}, s_{o}\right)}{\partial \theta_{o}} \cos \theta_{o}^{*}+\psi_{o}\left(\theta_{o}^{*}, s_{o}\right) \sin \theta_{o}^{*}\right)\right. \\
\left.+\sigma_{u o}\left(\frac{\partial f_{o}\left(\theta_{o}^{* *}, \sigma_{u o}, s_{o}\right)}{\partial \theta_{o}} \sin \theta_{o}^{* *}-f_{o}\left(\theta_{o}^{* *}, \sigma_{u o}, s_{o}\right) \cos \theta_{o}^{* *}\right)\right]^{2}>0 . \\
\forall 0 \leq \theta_{o}^{*}, \quad \theta_{o}^{* *} \leq \pi .
\end{gathered}
$$

If (3.11) is satisfied, then the operator $A$ is strictly monotone.

c. OPERATOR A IS CONTINUOUS BY LIPSCHITZ

We show that the operator $A$ has a continuous Gateau differential, i.e.

$$
(D A(u, h), v)_{H} \leq m(u)\|h\|_{H}\|v\|_{H}, \quad 0<m<+\infty .
$$

By definition

$$
D f\{a, b\}=\left.\frac{d}{d \xi} f(a+\xi b)\right|_{\zeta \rightarrow 0}=\frac{\partial f}{\partial a_{i j}} b_{i j}
$$

Applying to the operator $A$

$$
\begin{aligned}
(A u, v)_{H}= & \int_{\Omega}\left[-\frac{2}{3} \frac{\sigma_{u o} f_{o}}{\sin \theta_{o}} \dot{e}_{i j}(u)+\left(\psi_{o}+\sigma_{u o} f_{o} \cot \theta_{o}\right) \frac{S_{i j}}{\sigma_{u o}} v_{u o}(u)\right] e_{i j}(v) d \Omega \\
& +9 K \int_{\Omega} \dot{e}(u) \dot{e}(v) d \Omega
\end{aligned}
$$


we get:

$$
\begin{aligned}
(D A(u, h), v)_{H}=\int_{\Omega}[- & \frac{2}{3} \frac{\sigma_{u o} f_{o}}{\sin \theta_{o}} \delta_{i m} \delta_{j n}-\frac{2}{3} \frac{\partial}{\partial \theta_{o}}\left(\frac{\sigma_{u o} f_{o}}{\sin \theta_{o}}\right) \frac{\partial \theta_{o}}{\partial \dot{e}_{m n}} \dot{e}_{i j}(u) \\
& +\frac{\partial}{\partial \theta_{o}}\left(\psi_{o}+\sigma_{u o} f_{o} \cot \theta_{o}\right) \frac{\partial \theta_{o}}{\partial \dot{e}_{m n}} \frac{S_{i j}}{\sigma_{u o}} v_{u o}(u) \\
& \left.+\left(\psi_{o}+\sigma_{u o} f_{o} \cot \theta_{o}\right) \frac{S_{i j}}{\sigma_{u o}} \frac{\partial v_{u o}}{\partial \dot{e}_{m n}}\right] \dot{e}_{m n}(h) \dot{e}_{i j}(v) d \Omega \\
& +9 K \int_{\Omega} \dot{e}(h) \dot{e}(v) d \Omega .
\end{aligned}
$$

Since

$$
\frac{\partial v_{u o}}{\partial \dot{e}_{m n}}=\frac{2}{3 v_{u o}} \dot{e}_{m n}, \quad \frac{\partial \theta_{o}}{\partial \dot{e}_{m n}}=-\frac{1}{\sin \theta_{o}}\left(\frac{S_{m n}}{\sigma_{u o} v_{u o}}-\frac{2}{3} \cos \theta_{o} \frac{\dot{e}_{m n}}{v_{u o}^{2}}\right),
$$

we have:

$$
\begin{aligned}
(D A(u, h), v)_{H}= & \int_{\Omega}\left[-\frac{2}{3} \frac{\sigma_{u o} f_{o}}{\sin \theta_{o}} \delta_{l m} \delta_{j n}\right. \\
& +\frac{2}{3} \frac{\sigma_{u o}}{\sin \theta_{o}} \frac{\partial}{\partial \theta_{o}}\left(\frac{f_{o}}{\sin \theta_{o}}\right) \dot{e}_{u j}(u)\left(\frac{S_{m n}}{\sigma_{u o} v_{u o}}-\frac{2}{3} \cos \theta_{o} \frac{\dot{e}_{m n}(u)}{v_{u o}^{2}}\right) \\
& -\frac{1}{\sin \theta_{o}} \frac{\partial}{\partial \theta_{o}}\left(\psi_{o}+\sigma_{u o} f_{o} \cot \theta_{o}\right) \frac{S_{i j}}{\sigma_{u o}}\left(\frac{S_{m n}}{\sigma_{u o}}-\frac{2}{3} \cos \theta_{o} \frac{\dot{e}_{m n}(u)}{v_{u o}}\right) \\
& \left.+\left(\psi_{o}+\sigma_{u o} f_{o} \cot \theta_{o}\right) \frac{2}{3} \frac{S_{i j}}{\sigma_{u o}} \frac{\dot{e}_{m n}(u)}{v_{u o}}\right] \cdot \dot{e}_{m n}(h) \dot{e}_{i j}(v) d \Omega \\
& +9 K \int_{\Omega} \dot{e}(h) \dot{e}(v) d \Omega \\
\leq & \int_{\Omega}\left\{\left|\frac{\sigma_{u o} f_{o}}{\sin \theta_{o}}\right|+\left|1+\cos \theta_{o}\right|\left|\frac{\sigma_{u o}}{\sin \theta_{o}} \frac{\partial}{\partial \theta_{o}}\left(\frac{f_{o}}{\sin \theta_{o}}\right)\right|\right. \\
& +\left|1+\cos \theta_{o}\right|\left|\frac{1}{\sin \theta_{o}} \frac{\partial}{\partial \theta_{o}}\left(\psi_{o}+\sigma_{u o} f_{o} \cot \theta_{o}\right)\right| \\
& +9 K \int_{\Omega} \dot{e}(h) \dot{e}(v) d \Omega \\
\leq & m\left[\int_{\Omega} \frac{2}{3} v_{u o}(h) v_{u o}(v) d \Omega+3 \int_{\Omega} \dot{e}(h) \dot{e}(v) d \Omega\right]
\end{aligned}
$$


where

$$
\begin{aligned}
m=\max _{u}\left\{\frac { 2 } { 3 } \left(\left|\frac{\sigma_{u o} f_{o}}{\sin \theta_{o}}\right|+\left|\psi_{o}+\sigma_{u o} f_{o} \cot \theta_{o}\right|\right.\right. \\
+\left|1+\cos \theta_{o}\right|\left|\frac{\sigma_{u o}}{\sin \theta_{o}} \frac{\partial}{\partial \theta_{o}}\left(\frac{f_{o}}{\sin \theta_{o}}\right)\right| \\
\left.\left.+\left|1+\cos \theta_{o}\right|\left|\frac{1}{\sin \theta_{o}} \frac{\partial}{\partial \theta_{o}}\left(\psi_{o}+\sigma_{u o} f_{o} \cot \theta_{o}\right)\right|\right) ; 3 K\right\} .
\end{aligned}
$$

Thus

$$
\begin{aligned}
&(D A(u, h), v)_{H} \leq m {\left[\left(\int_{\Omega} \frac{3}{2} v_{u o}^{2}(h) d \Omega\right)^{1 / 2}\left(\int_{\Omega} \frac{3}{2} v_{u o}^{2}(v) d \Omega\right)^{1 / 2}\right.} \\
&\left.+\left(\int_{\Omega} 3 \dot{e}^{2}(h) d \Omega\right)^{1 / 2}\left(\int_{\Omega} 3 \dot{e}^{2}(v) d \Omega\right)^{1 / 2}\right] \\
& \leq m\left(\int_{\Omega} \dot{e}_{i j}(h) \dot{e}_{i j}(h) d \Omega+3 \int_{\Omega} \dot{e}^{2}(h) d \Omega\right)^{1 / 2} \\
& \times\left(\int_{\Omega} \dot{e}_{i j}(v) \dot{e}_{i j}(v) d \Omega+\int_{\Omega} 3 \dot{e}^{2}(v) d \Omega\right)^{1 / 2}
\end{aligned}
$$

We obtain

$$
(D A(u, h), v)_{H} \leq m\|h\|_{H}\|v\|_{H},
$$

with the condition:

$$
\begin{aligned}
& 0<\left|\frac{\sigma_{u o} f_{o}}{\sin \theta_{o}}\right|+\mid \psi_{o}+ \sigma_{u o} f_{o} \cot \theta_{o}|+| 1+\cos \theta_{o}|| \frac{\sigma_{u o}}{\sin \theta_{o}} \frac{\partial}{\partial \theta_{o}}\left(\frac{f_{o}}{\sin \theta_{o}}\right) \mid \\
&+\left|1+\cos \theta_{o}\right|\left|\frac{1}{\sin \theta_{o}} \frac{\partial}{\partial \theta_{o}}\left(\psi_{o}+\sigma_{u o} f_{o} \cot \theta_{o}\right)\right|<+\infty \\
& 0 \leq \theta_{o} \leq \pi
\end{aligned}
$$

Finally, if the conditions (3.3), (3.7), (3.11) and (3.12) are satisfied, then the operator $A$ exists and has the following properties: coercivity, strict monotonicity and Lipschitz continuity. According to the Minty-Browder theorem [9], the operator equation (3.6) has a unique solution.

THEOREM. If the material functions $f_{o}, \psi_{o}$ satisfy the conditions (3.3), (3.7), (3.11), (3.12), then there exists a unique solution of the boundary value problem of the local theory of elastoplastic deformation processes (2.7) - (2.12). 


\section{Verification of the conditions (3.3), (3.7), (3.11) and (3.12)}

According to (1.2) material functions are of the form

$$
\begin{gathered}
f_{o}\left(\theta_{o}, \sigma_{u o}, s_{o}\right)=-\frac{1}{s_{o}} \sin \theta_{o}\left[1+\left(\frac{3 G s_{o}}{\sigma_{u o}}-1\right)\left(\frac{1-\cos \theta_{o}}{2}\right)^{\alpha}\right], \\
\psi_{o}\left(\theta_{o}, s_{o}\right)=\phi^{\prime}\left(s_{o}\right) \cos \theta_{o}-\left(3 G-\phi^{\prime}\left(s_{o}\right)\right)\left(\frac{1-\cos \theta_{o}}{2}\right)^{\beta} ; \\
\alpha \geq 1, \quad \beta>1, \quad 0 \leq \theta_{o} \leq \pi
\end{gathered}
$$

These functions $f_{o}$ and $\psi_{o}$ describe vectorial and scalar behaviours of materials under complex loading [2], the function $f$ expresses a local instantaneous turning rate of the stress vector in the direction of tangent to the strain trajectory and the function $\psi$ an increment of the stress intensity. Consider some of their properties:

$$
\begin{gathered}
\sigma_{u o}, s_{o} \text { are fixed and limited; } \\
\sigma_{u o}>0, \quad s_{o}>0 \\
0<\phi^{\prime}\left(s_{o}\right)<3 G, \quad \sigma_{u o} / s_{o}>\phi^{\prime}\left(s_{o}\right) \\
f_{o} \leq 0 \quad \text { for all } \theta_{o} \\
0 \leq \theta_{o} \leq \pi \quad \text { and } \quad\left|f_{o}\right| \leq C_{1}<+\infty .
\end{gathered}
$$

Since

$$
\frac{f_{o}}{\sin \theta_{o}}=-\frac{1}{s_{o}}\left[1+\left(\frac{3 G s_{o}}{\sigma_{u o}}-1\right)\left(\frac{1-\cos \theta_{o}}{2}\right)^{\alpha}\right]
$$

and

$$
\frac{\partial}{\partial \theta_{o}}\left(\frac{f_{o}}{\sin \theta_{o}}\right)=-\frac{\alpha \sin \theta_{o}}{2 s_{o}}\left(\frac{3 G s_{o}}{\sigma_{u o}}-1\right)\left(\frac{1-\cos \theta_{o}}{2}\right)^{\alpha-1},
$$

we obtain

$$
\begin{gathered}
\left|\frac{\partial f_{o}}{\partial \theta_{o}}\right| \leq C_{2}<+\infty ; \quad\left|\frac{f_{o}}{\sin \theta_{o}}\right| \leq C_{3}<+\infty \\
\left|\frac{1}{\sin \theta_{o}} \frac{\partial}{\partial \theta_{o}}\left(\frac{f_{o}}{\sin \theta_{o}}\right)\right| \leq C_{4}<+\infty .
\end{gathered}
$$


Similarly

$$
\begin{gathered}
\psi_{o} \geq 0 \text { with } 0 \leq \theta_{o} \leq \bar{\theta}_{o}<\frac{\pi}{2} \\
\psi_{o} \leq 0 \quad \bar{\theta}_{o} \leq \theta_{o} \leq \pi \\
\left|\psi_{o}\right| \leq M_{1}<+\infty
\end{gathered}
$$

Hence

$$
\left|\frac{\partial \psi_{o}}{\partial \theta_{o}}\right| \leq M_{2}<+\infty, \quad\left|\frac{1}{\sin \theta_{o}} \frac{\partial \psi_{o}}{\partial \theta_{o}}\right| \leq M_{3}<+\infty .
$$

Consequently, (3.3) and (3.12) are completely satisfied. Now consider (3.7) obtaining

$$
\begin{aligned}
B \equiv \psi_{o} \cos \theta_{o}-\sigma_{u o} f_{o} \sin \theta_{o} & \\
=\phi^{\prime}\left(s_{o}\right) & \cos ^{2} \theta_{o}-\left(3 G-\phi^{\prime}\left(s_{o}\right)\right) \cos \theta_{o}\left(\frac{1-\cos \theta_{o}}{2}\right)^{\beta} \\
& +\frac{\sigma_{u o}}{s_{o}} \sin ^{2} \theta_{o}\left[1+\left(\frac{3 G s_{o}}{\sigma_{u o}}-1\right)\left(\frac{1-\cos \theta_{o}}{2}\right)^{\alpha}\right] .
\end{aligned}
$$

Put $X=\cos \theta_{o}$; rewrite

$$
\begin{aligned}
& B(X)=\phi^{\prime}\left(s_{o}\right) X^{2}-\left(3 G-\phi^{\prime}\left(s_{o}\right)\right) X\left(\frac{1-X}{2}\right)^{\beta} \\
&+ \frac{\sigma_{u o}}{s_{o}}\left(1-X^{2}\right)\left[1+\left(\frac{3 G s_{o}}{\sigma_{u o}}-1\right)\left(\frac{1-X}{2}\right)^{\alpha}\right] \\
&-1 \leq X \leq 1 .
\end{aligned}
$$

We can show that $B(X)>0$ for $-1 \leq X \leq 1$ and

$$
\min _{-1 \leq X \leq 1} B(X)=B(1)=\phi^{\prime}\left(s_{o}\right)>0 .
$$

Hence $B \geq \phi^{\prime}\left(s_{o}\right)>0$. The conditions (3.11) can be verified similarly.

We also verify previous conditions by graphic consideration, based on experimental data of certain materials.

In particular cases, these conditions reduce to simpler form. For example, in the process of average curvature,

$$
f_{o}=-\frac{1}{s_{o}} \sin \theta_{o}, \quad \psi_{o}=\phi^{\prime}\left(s_{o}\right) \cos \theta_{o},
$$


and the previous conditions reduce to

$$
1 / s_{o}>0, \quad \phi^{\prime}\left(s_{o}\right)>0 .
$$

Naturally, the first condition is satisfied, since arc length of the deformation trajectory is positive and the second condition means that the material must be plastic strain-hardening. For perfectly plastic material there can be different strain states respected to one stress state.

\section{Conclusions}

1. The complete form of constitutive relations of the local theory of elastoplastic deformation processes is given. Material functions have been shown in (1.2). This stress-strain relationship is adequate to describe various complex deformation processes in the body.

2. The boundary value problem of local theory of elastoplastic deformation processes has a unique solution. The material functions satisfy the required conditions of the theorem.

\section{Acknowledgements}

The author would like to thank Professor W.S. Perriman and the colleagues of the School of Mathematics and Statistics, Curtin University of Technology for helping him to complete this work.

\section{References}

[1] H. B. Dao, "On the hypothesis of local determinancy in plasticity theory", Bulletin of the Moscow State University 2 (1965) 67-75, in Russian.

[2] H. B. Dao, "Some properties of functions in fundamental stress-strain relations. Selection 'applied solidity and plasticity"', Gorky USSR 17 (1981) 37-45, in Russian.

[3] H. B. Dao, "Uniqueness theorem of boundary value problem of plasticity using the hypothesis of local determinancy", Mechanics of Solids 17 (1982) 110-115, translated by Allerton Press Inc.

[4] H. B. Dao, "Research of the local theory of elastoplastic deformation processes", Dr Sc. Thesis, The Moscow State University, 1987, in Russian.

[5] A. A. Ilyushin, "On the stress-strain relationship in mechanics of continuum media", $J$. of Applied Mathematics and Mechanics 18 (1954) 641-666, in Russian. 
[6] V. S. Lensky, "Analysis of plastic behaviour of metals under complex loading. Plasticity", Proc. 2nd Symposium on naval structural mechanics, New York (1960) 259-278.

[7] V. S. Lensky, "Hypothesis of local determinancy in plasticity theory", Transactions of USSR Academy of Sciences 5 (1962) 154-158, in Russian.

[8] V. S. Lensky and E. V. Lensky, "Three-terms relation of general plasticity", Mechanics of Solids 20 (1985) 111-115.

[9] G. J. Minty, "Monotone (nonlinear) operators in hilbert space", Duke Math. J. 29 (1962) 341-346.

[10] E. Tanaka, "Hypothesis of local determinancy for five dimensional strain trajectories", Acta mechanica 52 (1984).

[11] M. Tokuda, Y. Ohashi et al., "On the hypothesis of local determinancy and a concise stress-strain relation for curved strain path", Bulletin of JSME 26 (1983) 1475-1480. 